\title{
Analysis of genome-wide association study data using the protein knowledge base
}

\author{
Sara Ballouz ${ }^{1,2}$, Jason Y Liu', Martin Oti ${ }^{3}$, Bruno Gaeta ${ }^{2}$, Diane Fatkin ${ }^{4,5}$, Melanie Bahlo ${ }^{6}$ and Merridee A Wouters ${ }^{7 *}$
}

\begin{abstract}
Background: Genome-wide association studies (GWAS) aim to identify causal variants and genes for complex disease by independently testing a large number of SNP markers for disease association. Although genes have been implicated in these studies, few utilise the multiple-hit model of complex disease to identify causal candidates. A major benefit of multi-locus comparison is that it compensates for some shortcomings of current statistical analyses that test the frequency of each SNP in isolation for the phenotype population versus control.

Results: Here we developed and benchmarked several protocols for GWAS data analysis using different in-silico gene prediction and prioritisation methodologies. We adopted a high sensitivity approach to the data, using less conservative statistical SNP associations. Multiple gene search spaces, either of fixed-widths or proximity-based, were generated around each SNP marker. We used the candidate disease gene prediction system Gentrepid to identify candidates based on shared biomolecular pathways or domain-based protein homology. Predictions were made either with phenotype-specific known disease genes as input; or without a priori knowledge, by exhaustive comparison of genes in distinct loci. Because Gentrepid uses biomolecular data to find interactions and common features between genes in distinct loci of the search spaces, it takes advantage of the multi-locus aspect of the data.

Conclusions: Results suggest testing multiple SNP-to-gene search spaces compensates for differences in phenotypes, populations and SNP platforms. Surprisingly, domain-based homology information was more informative when benchmarked against gene candidates reported by GWA studies compared to previously determined disease genes, possibly suggesting a larger contribution of gene homologs to complex diseases than Mendelian diseases.
\end{abstract}

\section{Background}

The identification of genes implicated in human disease enables an understanding of disease mechanisms and is essential for the development of diagnostics and therapeutics. Many associations have now been identified from GWA studies. As of September 2011, the HuGE database http://www.hugenavigator.net contained 6164 associations from 1019 published GWAS. These methods have led to the discovery of several novel genes for complex diseases. However GWAS have not proved as powerful as originally hoped with approximately 3061 genes reported or identified, suggesting more may be gleaned by careful reanalysis of the data.

\footnotetext{
* Correspondence: m.wouters@deakin.edu.au

${ }^{7}$ School of Life and Environmental Sciences, Deakin University, Geelong, VIC, 3217, Australia

Full list of author information is available at the end of the article
}

GWAS are designed to identify common genetic risk factors of complex diseases and quantitative traits, that are believed to be the result of multiple genetic and environmental factors [1]. GWAS use high-throughput genotyping platforms, such as SNP chips, which carry hundreds of thousands of SNP markers. Even with multiple marker testing, GWAS have greater statistical power to detect genetic variants that increase disease risks than linkage analysis [2], but hundreds of SNPs may be identified. In order to make sense of the large amount of data acquired, most published GWAS list only the top 20 to 50 most significant SNPs and their nearest gene using the "most significant SNPs/genes" approach [3], while ignoring the remaining SNPs [4-6]. A highly stringent significance threshold attempts to correct for the number of false positives, but this conservative statistical approach combined with the
Ciomed Central

다 2011 Ballouz et al; licensee BioMed Central Ltd. This is an Open Access article distributed under the terms of the Creative Commons Attribution License (http://creativecommons.org/licenses/by/2.0), which permits unrestricted use, distribution, and reproduction in any medium, provided the original work is properly cited. 
selection of the nearest-neighbouring gene to the significant SNP still has several limitations.

Phenotypes influenced by multiple genetic and environmental factors, or those with uncommon and small effect variants, are not detected after adjustment for multiple testing [7], thereby introducing a potentially high false negative rate to the study. Also, variants with larger effects might not always rank among the top markers reported when taking the most-significant SNP approach $[7,8]$.

A further conundrum for GWAS has been the lack of genetic signals recovered to explain the genetic heritability of many diseases, implying that much has been missed due to the limitations of GWAS methodology [9]. This missing heritability may be due to the heterogeneous population studied, for example the disease may be caused by multiple rare variants; the fact that SNPs are tested in isolation, for example if important gene-gene interactions occur; inability to control the environment of the patient population, for example gene-environmental interactions may be important; or gaps in SNP chip coverage for some regions of the genome.

Furthermore, tagged SNPs on the platforms used in the studies are potentially only in linkage disequilibrium (LD) with the causal SNPs and further replication studies and sequencing is required to identify the actual causal variant. With the advent of next-generation sequencing, rapid follow up of multiple candidate markers or genes is possible.

The methods typically used by researchers to select genes associated with the significant SNP assume the disease-associated SNP is either resident in, or adjacent to, the disease gene. But the genetic architecture of the genome is still not well understood: work on long range gene regulation [10] suggests distal cis-acting elements can control genes that are not directly adjacent to the regulatory region of the gene. For instance fibroblast growth factor $8, F G F 8$, is controlled by regulatory elements within and beyond the neighbouring gene FBXW4 [11]. Therefore, the disease gene may be near the significant or causal SNP but may not be the closest gene to it, i.e. the causal SNP is in a regulatory region that acts distally on the disease-causing gene. In these cases, the simplistic approach currently used for SNPto-gene mapping is limiting and the search space should be extended to include additional nearby genes that may play a role in the phenotype.

Clearly new approaches are required to utilize the valuable but noisy data from GWAS. In order to avoid "throwing the baby out with the bathwater", the statistical significance threshold can be decreased to study a much larger sample of SNPs which may potentially be associated with the disease. Although, this reduces the power of the study, these less significant SNPs can then be sifted using other information. Several approaches have been suggested. Genetic information can be used to "weight" SNPs according to their plausibility. Information can be in the form of genome-wide linkage from population data $[12,13]$; or prior probabilities of association in significance calculations $[14,15]$. In addition to genetic data, biomolecular data such as information on protein function and protein-protein interactions can provide valuable information to distinguish associated loci from noise.

Gentrepid is a second generation candidate gene prediction system tool that draws on two types of functional data to group genes [16,17]. The Common Pathway Scanning (CPS) module is a Systems Biology method based on the assumption that common phenotypes are likely to be associated with proteins that partake in the same complex or pathway [18]. In other words, disease-causing genes for a specific phenotype are more likely to interact with other phenotype-specific disease genes $[19,20]$. Potential disease genes are predicted by identifying all proteins within phenotype-associated loci that are part of a pathway or complex.

The second Gentrepid module is Common Module Profiling (CMP), a technique based on the principle that candidate genes have similar functions to disease genes already determined for the phenotype [21]. A unique feature of CMP is that it uses domain-based comparative sequence analysis to identify proteins with potential functional similarity. In the field of candidate disease gene prediction, genes are often treated as single functional units, but translated proteins fold into discrete globular structures of limited size called domains [22]. Sequence-determined autonomous folding of domains into conserved compact three-dimensional structures is proposed to occur through hydrophobic collapse. A domain-based sequence comparison approach has several advantages over protein-based ones. Multiple domains, each with their own biochemical function, are often combined into a single gene to encode its entire function in a modular fashion similar to LEGO blocks [23-25]. Dissection of a gene into domains thus potentially provides a more fine-grained approach to functional assignment than can be achieved on a gene-bygene basis. In addition, a particular isoform of a gene may be implicated. At the phenotypic level, functional clustering can be used to advantage as there are fewer building blocks than genes. The number of human genes stands at 25,000 to 35,000 [26], and the estimated size of the proteome ranges from 90,000 [27] to $1,000,000$ [28]. In comparison, the fold repertoire of domains in the planetary proteome has been estimated at between 1000 and 5000 folds [29-31]. A domainbased approach also enables better detection and 
annotation of protein features. Because structure is conserved over sequence, domain-based sequence comparison searches have been shown to be more accurate than full-sequence searches [32]. Using the Pfam library of Hidden Markov models [29], domains can be assigned to approximately $69 \%$ of human proteins which allows functional inference for around 54\% of the human genome. Prioritisation of the genes predicted from the modules is based on the statistical significance of the results.

Here we developed and benchmarked several protocols for analysing GWAS data effectively using the wellstudied WTCCC data set on seven diseases. This data is employed in two manners: firstly using known disease genes for a particular phenotype to seed the search; and secondly using an agnostic approach which searches for de novo relationships between multiple loci. Predictions are then benchmarked against known disease genes, and genes suggested by the WTCCC study. The results show that analysis of more SNPs and consideration of more genes around each SNP replicate data from previous studies more effectively. The system was capable of extracting significantly associated genes from those of lower significance, as well as known and novel candidate disease genes using either a priori genetic knowledge or de novo analysis.

\section{Results}

To test the ability of Gentrepid to select and prioritise valid disease gene candidates from the SNPs of GWAS, we performed a series of analyses on data from casecontrol studies from the WTCCC [33]. Most early GWAS used the Affymetrix chip set with approximately 500,000 known SNPs (Affy500k). We extracted 459,231 autosomal SNPs from the chip set for further analysis as detailed in the methods.

\section{Average number of SNP associations per phenotype}

First we selected appropriate significance thresholds for GWA SNPs that are associated with the phenotypes of interest by increasing the cut-off of the Cochran-Armitage association $p$-values. Although the data quality varies depending on the phenotype, four consistent thresholds were used for ease of comparison: a weakly significant set (WS, $p_{G W A} \leq 10^{-3}$ ), a moderately-weak significant set (MWS, $p_{G W A} \leq 10^{-4}$ ), a moderately-high significant set (MHS, $P_{G W A} \leq 10^{-5}$ ), and a highly significant SNP set $\left(\mathrm{HS}, p_{G W A}<5 \times 10^{-7}\right)$. Table 1 summarizes the average number of SNPs above each of the significance thresholds that were associated with the phenotypes. On average, 30 highly significant SNPs were associated with a phenotype and this rose to over 800 SNPs for the weakly significant data. We then clustered co-located SNPs into what we termed an "associated locus" (See Methods). Significant SNPs show strong
Table 1 Average number of SNPs, loci and genes per phenotypes used in this study with significant association $p$ values and associated annotated genes in Gentrepid

\begin{tabular}{|c|c|c|c|c|c|c|}
\hline & & & \multicolumn{4}{|c|}{ Level } \\
\hline & & & WS & MWS & MHS & HS \\
\hline & & & $p \leq{ }_{3}^{1 e-}$ & $p \leq \underset{4}{1 \mathrm{e}-}$ & $p \leq{ }_{5}^{1 e-}$ & $\begin{array}{c}p<5 e- \\
7\end{array}$ \\
\hline SNPs & & & 804.29 & 160.29 & 56.71 & 29.14 \\
\hline Loci & & & 446.86 & 84.43 & 18.71 & 7.29 \\
\hline \multirow[t]{6}{*}{ Total Genes } & $B Y$ & $1 \mathrm{Mbp}$ & 3875.57 & 870.86 & 175.29 & 87.43 \\
\hline & & $0.5 \mathrm{Mbp}$ & 2140.00 & 477.29 & 106.00 & 57.29 \\
\hline & & $0.1 \mathrm{Mbp}$ & 654.57 & 148.43 & 43.71 & 23.00 \\
\hline & $N N$ & Adjacent & 1412.14 & 292.43 & 62.29 & 26.14 \\
\hline & & Nearest & 452.86 & 91.00 & 22.29 & 10.14 \\
\hline & & Resident & 198.71 & 42.57 & 11.43 & 5.43 \\
\hline \multirow{6}{*}{$\begin{array}{l}\text { Annotated } \\
\text { Genes }\end{array}$} & $B Y$ & $1 \mathrm{Mbp}$ & 2285.29 & 528.86 & 116.43 & 61.57 \\
\hline & & $0.5 \mathrm{Mbp}$ & 1275.57 & 300.43 & 73.14 & 41.57 \\
\hline & & $0.1 \mathrm{Mbp}$ & 426.43 & 103.43 & 32.00 & 16.57 \\
\hline & NN & Adjacent & 803.14 & 172.00 & 40.71 & 17.57 \\
\hline & & Nearest & 285.71 & 59.00 & 15.57 & 6.14 \\
\hline & & Resident & 155.29 & 33.43 & 8.86 & 3.57 \\
\hline
\end{tabular}

Column abbreviations: HS, highly significant; MHS, moderately-high significance; MWS, moderately-weak significance; WS, weakly significant. Row abbreviations: SNPs, average number of implicated SNPs per phenotype; Loci, average number of SNP clusters per phenotype; "Total Genes", the average number of genes per phenotype in the designated constructed pseudointervals; "Annotated Genes", the average number of genes per phenotype in the designated constructed pseudo-intervals with Gentrepid annotations.

clustering, with $50-60 \%$ of significant SNPs clustering in phenotype-specific loci, with an average of 3 SNPs per cluster. The HS threshold had, on average, 7 associated loci per phenotype whereas the average number of associated loci for the WS threshold was over 400 (Table 1).

\section{Gene selection around associated loci}

To further investigate the relationship between phenotype-associated loci and nearby genes, genes were selected within a series of pseudo-intervals constructed around loci using one of two major assumptions (Figure 1 ). The first assumption, which gathers genes based on proximity to the associated locus, we termed the Nearest Neighbour (NN) approach. To enable discovery of genes subject to longer range regulation, we adopted an additional distance-based Bystander (BY) approach whereby genes were captured from an interval of fixed size created around each locus. For the NN approach, three sets of genes were created: a set containing genes with loci internal to the gene termed the resident set; a second set with loci directly adjacent to the gene, termed the nearest set; and a third set with the loci either resident in, or directly adjacent to, the four nearest genes, termed the adjacent set (Figure 1). NN sets 


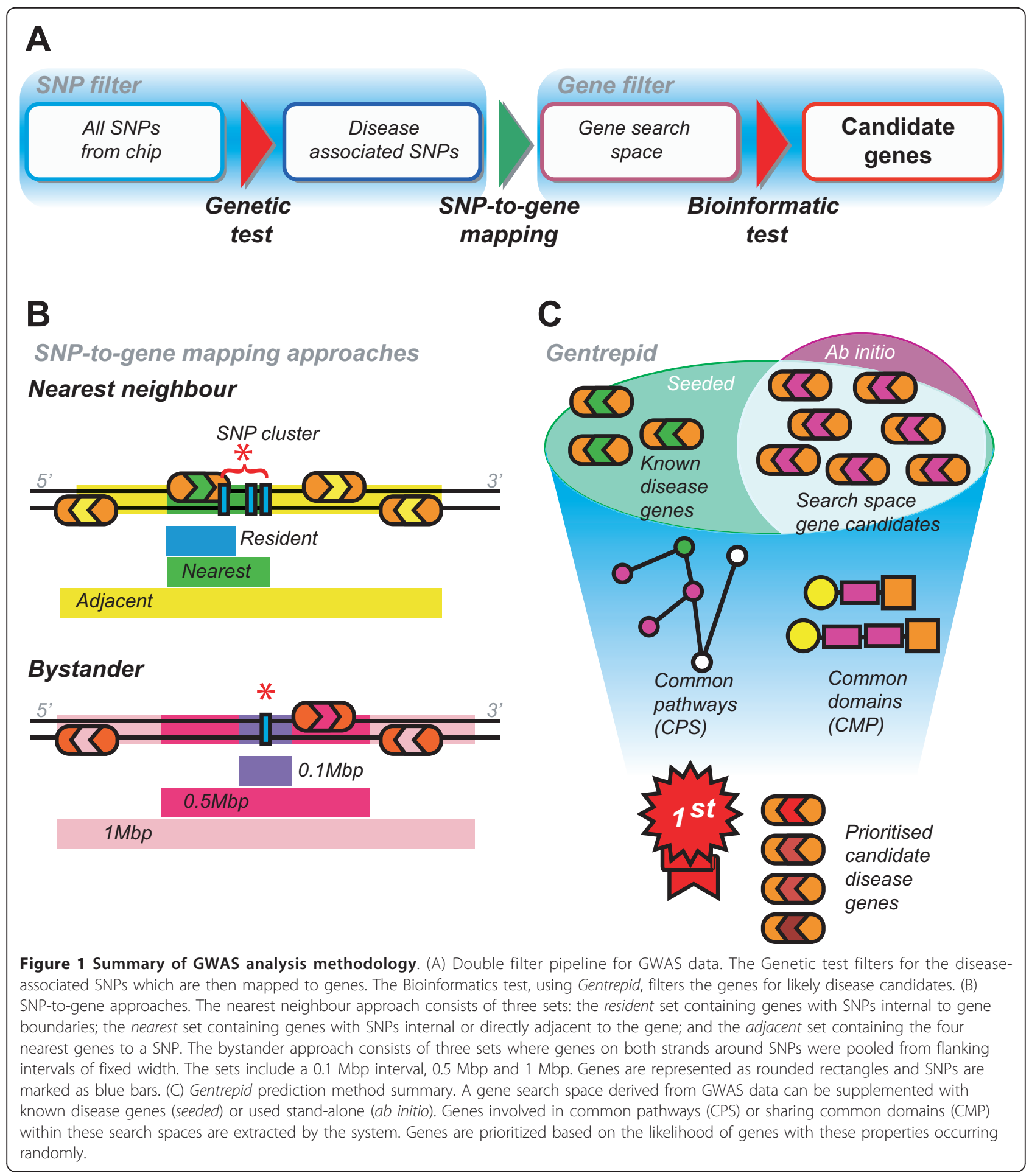

are not distance based. For the BY approach, three pseudo-intervals of different sizes were tested: genes were pooled from flanking intervals of $0.1 \mathrm{Mbp}, 0.5$ Mbp or $1 \mathrm{Mbp}$ in width around loci (Figure 1).

The 24 implicated search spaces per phenotype constructed using multiple SNP significance thresholds and gene selection methods ranged in size from 2 to 4431 genes: up to $10 \%$ of the genome. We have previously shown that candidate gene prediction by Gentrepid in such large search spaces is computationally feasible [17]. As shown in Table 1, more genes are associated with the phenotype-specific loci in the two larger bystander 
intervals (0.5 Mbp and $1 \mathrm{Mbp})$. However, the adjacent NN gene set usually contains more genes than the smallest BY interval for each phenotype $(0.1 \mathrm{Mbp})$, as often one of the adjacent genes is located farther than the 50 $\mathrm{Kbp}$ distance threshold used $(0.1 \mathrm{Mbp} / 2)$. Genes in the adjacent set are on average $362 \mathrm{Kbp}(178-388 \mathrm{Kbp})$ away from the associated SNP, whilst genes in the nearest set are on average $90 \mathrm{Kbp}$ (20-96 Kbp) away.

\section{Constraints on genomic coverage}

As a baseline, we wished to establish how genomic coverage by the Affy500K SNP chip set used in the WTCCC study depended on the approaches and assumptions used, and if these genes were represented in Gentrepid by associated pathways and domains. Figure 2 shows coverage of the human genome by the Affy500K chip set using the three gene selection methods for each of the NN and BY approaches tested. Here we define genes that are present in RefSeq [26] as "characterized" genes and those that have either a domain predicted through Pfam [29], or pathways and interactions partners in Gentrepid as "annotated". By selecting only the nearest gene to the associated SNP, as currently done in GWAS (nearest NN set), only 76\% of characterized genes are associated with a SNP. Gene coverage increases to $90 \%$ if we associate nearest genes in the 3' and 5' direction on both strands with the SNP (adjacent NN set). Using the BY approach, gene coverage increases with interval size, ranging from 96\% (0.1 Mbp) to $99.4 \%$ (1 Mbp) of characterized genes. SNPs on the Affy500K chip sets are randomly distributed across the genome, and ignore LD patterns. We confirmed this by observing that the density of SNPs on the chip sets is similar to the density of SNPs across the genome from dbSNP (data not shown). The Affy500K chip set's coverage of the genome has been shown to be poorer than other platforms that attempt to capture as much genetic variation as possible through LD-based tagging [34].

\section{Comparative overview of candidate gene predictions}

To assess the ability of the two Gentrepid modules (CPS and CMP) to independently extract positional candidates from less significant data, we analysed the GWAimplicated pseudo-intervals chosen using both the NN and BY assumptions at the different levels of stringency. Two modes of input, referred to as "seeded mode" and "ab initio mode" were used to determine the common properties of phenotype-specific genes within the six gene sets for each disease. Seeded mode is assisted by phenotype-associated genes from OMIM [35] (Additional file 1, Table S1). The "known" disease genes were defined as those determined prior to GWAS of these

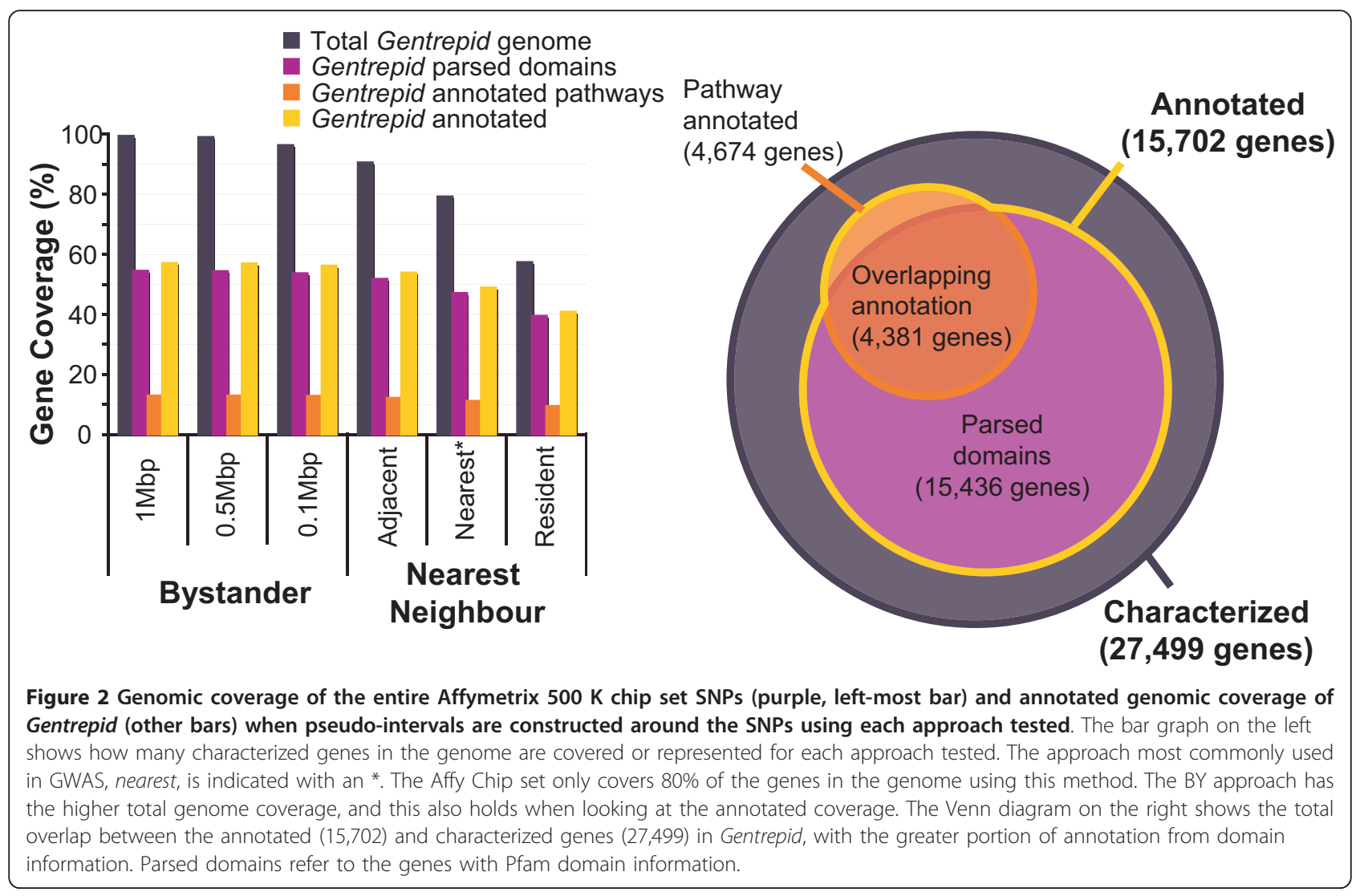


diseases, and therefore are restricted to OMIM entries. $A b$ initio mode uses only genes pooled from the SNPassociated intervals: no additional genetic data beyond the GWA-implicated loci is required.

We compared the average number of significant predictions made by each of the modes and modules and the complementarity of these predictions (Table 2) across all search spaces tested. A CPS prediction was considered significant at $p<0.05$, whilst CMP predictions were determined through scores/statistical tests described in detail in the methods section. Ab initio mode had, on average, more predictions when compared to seeded mode for the same gene search space set, indicating that there was novel information in the genetic data that was not represented by the known disease genes: a success for the GWAS methodology. In some instances, there were no predictions made by the seeded mode, suggesting earlier studies were either on the wrong track, or their results are limited to the studied family. Interesting differences were noted between the two bioinformatic methods depending on the significance of the SNPs used. Fewer predictions were made in the nearest and resident approaches of the HS and MHS thresholds, suggesting that the use of more generous thresholds may be detecting weaker effects. CPS seeded made more predictions than CMP seeded. CPS $a b$ initio made, on average, more predictions than CMP $a b$ initio for the WS and MWS data. For the MHS and HS thresholds, CMP ab initio made more predictions than CPS $a b$ initio except for the HS nearest set where CMP $a b$ initio made no predictions. For the stringent thresholds, the number of predictions was similar for each module.

A summary of the proportion of the total number of significant predictions made by each module is represented in Figure 3. For seeded predictions, CPS made more predictions than CMP, as shown by the distinct data points on the left hand side of Figure 3. CPS predicted between $59-100 \%$ of total seeded predictions, while CMP only predicted between $12-43 \%$ of total seeded predictions. However, at most $12 \%$ of these predictions are common to the two modules (joined diamonds at bottom). In the $a b$ initio analysis, CMP made a larger contribution to the number of predictions compared to the seeded mode. CPS predicted between 14$100 \%$ of $a b$ initio predictions, while CMP predicted between $27-88 \%$ of $a b$ initio predictions. But again, the two modules were relatively independent with $12 \%$ of these predictions in common. The percentage of $a b$ initio predictions made by each module varied

Table 2 Average number of gene predictions made by Gentrepid modes and modules

\begin{tabular}{|c|c|c|c|c|c|c|c|c|c|c|c|c|c|c|}
\hline \multirow{3}{*}{$\begin{array}{l}\text { Mode } \\
\text { Seeded }\end{array}$} & \multirow{2}{*}{\multicolumn{2}{|c|}{$\begin{array}{l}\text { SNP/gene } \\
\text { Approach }\end{array}$}} & \multirow{2}{*}{$\frac{\text { CPS }}{\text { WS }}$} & \multirow[b]{2}{*}{ MWS } & \multirow{3}{*}{$\frac{\text { MHS }}{2.29}$} & \multirow{2}{*}{\multicolumn{2}{|c|}{$\frac{\text { CMP }}{\text { WS }}$}} & \multirow{3}{*}{$\frac{\text { MWS }}{3.86}$} & \multirow{3}{*}{$\frac{\text { MHS }}{0.71}$} & \multirow{3}{*}{$\frac{\text { HS }}{0.43}$} & \multicolumn{2}{|c|}{$\mathrm{CPS}+\mathrm{CMP}$} & \multirow[b]{2}{*}{ MHS } & \multirow{3}{*}{$\begin{array}{c}\text { HS } \\
1.14\end{array}$} \\
\hline & & & & & & & & & & & WS & MWS & & \\
\hline & $B Y$ & $1 \mathrm{Mbp}$ & 29.14 & 6.00 & & 0.71 & 15.43 & & & & 42.86 & 9.43 & 3.00 & \\
\hline & & $0.5 \mathrm{Mbp}$ & 12.29 & 5.29 & 2.00 & 1.29 & 9.00 & 2.86 & 0.57 & 0.29 & 20.71 & 7.71 & 2.57 & 1.57 \\
\hline & & $0.1 \mathrm{Mbp}$ & 6.71 & 3.00 & 1.71 & 1.00 & 3.14 & 1.00 & 0.29 & 0.00 & 9.57 & 3.57 & 1.86 & 1.00 \\
\hline & $N N$ & Adjacent & 19.14 & 6.00 & 1.57 & 1.43 & 6.86 & 1.57 & 0.43 & 0.00 & 25.29 & 7.00 & 1.86 & 1.43 \\
\hline & & Nearest & 10.86 & 2.57 & 1.43 & 1.00 & 3.00 & 0.86 & 0.29 & 0.00 & 13.71 & 3.29 & 1.57 & 1.00 \\
\hline & & Resident & 4.29 & 1.43 & 1.00 & 0.57 & 1.29 & 0.43 & 0.14 & 0.00 & 5.57 & 1.86 & 1.14 & 0.57 \\
\hline \multirow[t]{6}{*}{ Ab initio } & $B Y$ & $1 \mathrm{Mbp}$ & 105.00 & 27.14 & 6.86 & 5.86 & 57.57 & 14.71 & 12.57 & 10.14 & 157.14 & 40.71 & 17.43 & 14.29 \\
\hline & & $0.5 \mathrm{Mbp}$ & 41.29 & 17.43 & 5.86 & 2.86 & 30.71 & 12.71 & 8.57 & 10.43 & 70.00 & 29.57 & 14.43 & 11.86 \\
\hline & & $0.1 \mathrm{Mbp}$ & 28.57 & 6.71 & 2.00 & 0.57 & 12.14 & 10.00 & 7.86 & 3.57 & 38.14 & 16.71 & 9.86 & 4.14 \\
\hline & NN & Adjacent & 59.57 & 13.86 & 2.14 & 0.71 & 26.86 & 8.71 & 5.29 & 2.00 & 81.14 & 21.29 & 6.71 & 2.71 \\
\hline & & Nearest & 28.71 & 5.14 & 1.00 & 0.57 & 10.86 & 2.00 & 1.71 & 0.00 & 37.43 & 6.86 & 2.71 & 0.57 \\
\hline & & Resident & 13.00 & 2.57 & 0.29 & 0.00 & 9.71 & 0.86 & 0.57 & 0.00 & 21.00 & 3.14 & 0.86 & 0.00 \\
\hline \multirow[t]{6}{*}{ Seeded and ab initio } & BY & $1 \mathrm{Mbp}$ & 105.00 & 27.14 & 6.86 & 6.57 & 72.43 & 18.43 & 13.29 & 10.57 & 170.14 & 44.00 & 18.14 & 15.43 \\
\hline & & $0.5 \mathrm{Mbp}$ & 41.43 & 17.43 & 5.86 & 3.57 & 39.57 & 15.57 & 9.14 & 10.71 & 78.43 & 32.00 & 15.00 & 12.86 \\
\hline & & $0.1 \mathrm{Mbp}$ & 28.57 & 7.00 & 2.29 & 1.29 & 15.29 & 11.00 & 8.14 & 3.57 & 40.57 & 17.57 & 10.29 & 4.86 \\
\hline & NN & Adjacent & 59.57 & 13.86 & 2.29 & 1.71 & 33.43 & 10.29 & 5.71 & 2.00 & 86.57 & 22.29 & 7.14 & 3.71 \\
\hline & & Nearest & 28.71 & 5.29 & 1.71 & 1.14 & 13.86 & 2.86 & 2.00 & 0.00 & 39.86 & 7.57 & 3.57 & 1.14 \\
\hline & & Resident & 13.00 & 2.71 & 1.00 & 0.57 & 10.71 & 1.29 & 0.71 & 0.00 & 22.00 & 3.71 & 1.71 & 0.57 \\
\hline
\end{tabular}

Each cell represents the number of predictions averaged across the seven phenotypes made by the mode and module. Column abbreviations: HS, highly significant; MHS, moderately-high significance; MWS, moderately-weak significance; WS, weakly significant; CPS, common pathway scanning module; CMP, common module profiling. Row abbreviations: Seeded, mode using known disease gene information as seeds; $A b$ initio, blind approach mode; BY, bystander approach; NN, nearest neighbour approach. The total number of predictions made by Gentrepid for each significance threshold and SNP/gene approach are bolded. All results have been filtered on significance. For CPS, pathways reach a significance threshold of $p<0.05$ based on Fishers exact test. For CMP seeded, a threshold of 0.4. For CMP ab initio, a threshold of $\chi_{\text {max unique }}^{2}>105$ and $\chi^{2}$ min $>7.88$ for multidomain proteins and $\chi^{2}$ min $>100$ for single domain proteins. 


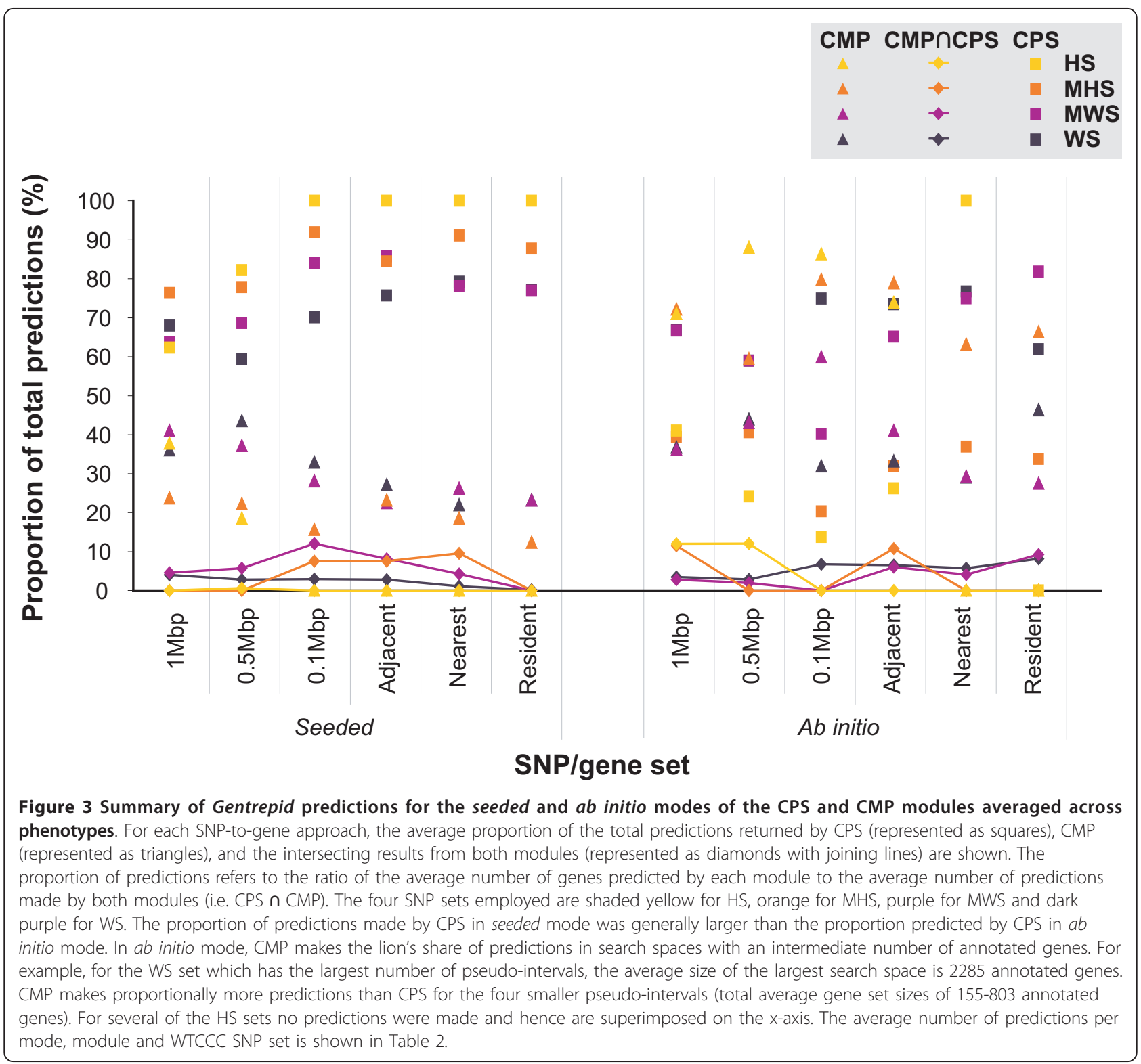

depending on the gene selection method and significance threshold, as shown by the less distinct separation of points on the right hand side of Figure 3. For the WS sets, CPS made more $a b$ initio predictions; while for the MHS and HS sets, CMP made more predictions except for the smaller nearest and resident sets. The fewer predictions by CMP ab initio could reflect one of two things: true biological signals in complex diseases are missed in these smaller sets because candidate genes with long range effects are not included, or that the predictions made in the smaller sets with fewer genes fail to reach significance in our tests. Seeded and $a b$ initio predictions are most congruent for CPS, with shared predictions comprising between $16-62 \%$ of total CPS predictions. For CMP, the predictions made by the seeded and ab initio modes are dissimilar, with 0-3\% overlap in predictions. The congruency in predictions between CPS seeded and ab initio indicates that CPS works well as a search tool because the same pathways are being selected by the $a b$ initio method without the $a$ priori knowledge supplied to the seeded method. CPS is also a good discovery tool as there are novel predictions made by $a b$ initio that were not detected by the seeded method. On the other hand, the fewer similar predictions made through the CMP seeded and ab initio modules might reflect differences in candidate gene significance filtering, or perhaps that CMP ab initio is making many more novel predictions and is a much stronger discovery tool for GWAS data. As the pathways utilized by CPS are often elucidated as part of the 
disease discovery process, the domain-based CMP approach may be a superior source of novel predictions for poorly characterised diseases when implemented using the agnostic ab initio approach.

\section{Benchmarking against validation sets}

As Gentrepid is intended as a discovery tool, there is currently no absolute way to determine if the candidate genes selected by the Gentrepid modules are indeed true positives without further genetic and molecular analyses on patients with the genotype. As a proxy, we tested Gentrepid on two datasets containing either known causal genes or genes that have a high probability of being causal. The first set consisted of known disease genes and loci from the phenotype data; whereas the second set of genes were implicated by the WTCCC as candidates. The ability of Gentrepid to extract and prioritise the genes in these sets was tested (Figure 4). In order to determine the overall performance of the modules being tested on the validation set, we calculated the sensitivity, specificity and enrichment ratios of each of the methods for each significance threshold (Figure 5).

Our first validation set consisted of 97 known disease genes collated from OMIM for the 7 diseases from the WTCCC study (Additional file 1, Table S1). Of these 97 genes, up to 29 were within WTCCC-implicated loci depending on the SNP-to-gene selection method employed and 7 of these genes were in highly significant loci (Additional file 1, Table S2). However the remaining known disease genes, constituting $70 \%$ of genes extracted from OMIM, were not in the search spaces at all. The lack of congruence between previous studies and the WTCCC data could be due to several factors which include differences in study design, differences in studied populations leading to allelic and locus heterogeneity, or true genetic differences. Our second validation set consisted of the WTCCC-implicated candidates, a total of 62 genes, from both significant and modestly associated SNPs [33] (Additional file 1, Table S1). Of these genes, 47 were within the search space, but only 39 were Gentrepid annotated genes. Of the 39 genes in at least one search space, 16 were in highly significant loci (Additional file 1, Table S3).

The ability of CPS to predict and prioritise known disease genes is shown in Figure 4A. A predicted gene is assigned an ordinal priority based on the statistical significance of the pathway it shares with other phenotypeimplicated genes and thus has a rank equal to other candidates in the same pathway. The majority of known disease genes were in the highest ranked pathway for the phenotype. Known disease genes comprised $62 \%$ of all seeded predictions and $42 \%$ of all $a b$ initio predictions. Most genes in the $0.1 \mathrm{Mbp}$ and adjacent approaches were ranked $1^{\text {st }}$, but generally the gene selection method used had little effect on priority. Some deterioration of the signal is apparent for the least statistically significant data (WS), when the more demanding $a b$ initio method is employed; or when larger search spaces are used. The ability of CPS to prioritise WTCCC phenotype-specific candidates is shown in Figure 4B. Despite being confronted with increasingly large search spaces, CPS is still able to extract biologically relevant genes from the increasingly less significant genetic data. Genes associated with the most statistically significant SNPs were primarily ranked first, constituting up to $66 \%$ of all predictions in seeded mode and $46 \%$ of all $a b$ initio predictions. Of the 16 annotated WTCCC candidates in the HS sets: 4 candidates from the adjacent set were predicted by CPS seeded and given the top priority; 3 genes were predicted and given the top priority by CPS $a b$ initio, and a fourth gene was ranked $2^{\text {nd }}$. Overall for CPS, genes in both validation sets were ranked first when the $0.1 \mathrm{Mbp}$ or adjacent gene selection methods were used.

The ability of CMP to prioritise known disease genes is shown in Figure 4C. A predicted gene is assigned an ordinal priority based on its score in CMP seeded, and the $\chi^{2}$ min score of CMP $a b$ initio. Only 7 pairs of the phenotype-specific known disease genes share common domains, so CMP seeded was not expected to make many predictions based on the available input. Even so, only a single known gene was predicted by CMP seeded, TCF2 for the T2D phenotype which shares hepatocyte nuclear factor 1 domains HNF-1B_C (PF04812) and HNF-1_N (PF04814) with known disease gene TCF1. CMP $a b$ initio predicted CARD15/NOD2 for the CD phenotype, but other predictions did not pass the required thresholds. The ability of CMP to prioritise WTCCC phenotype-specific candidates is shown in Figure 4D. CMP seeded only predicts HHEX for the T2D phenotype based on the homeobox domain it has in common with known disease genes IPF1 and PAX4. CMP $a b$ initio predicted a total of 6 of the 39 WTCCC candidates, ranking the predicted genes $1^{\text {st }}$ to $10^{\text {th }}$. Overall, CMP prioritised the WTCCC validation set genes in the top ten in a manner that was in rough agreement with SNP significance.

Further to the prioritisation results, the specificity, sensitivity, and the enrichment ratio (ER) metrics allow for an overall quantitative comparison of the performance of the individual modes and modules (Figure 5, Additional file 1, Table S4). The sensitivity of the system ranged between 0.09 and 1. CPS in both seeded and $a b$ initio mode had higher sensitivity scores compared to CMP. CPS ab initio generally had the highest sensitivity compared to the other modes and modules; holding true for both validation sets. Using the known WS set for validation, CPS seeded had a sensitivity that 


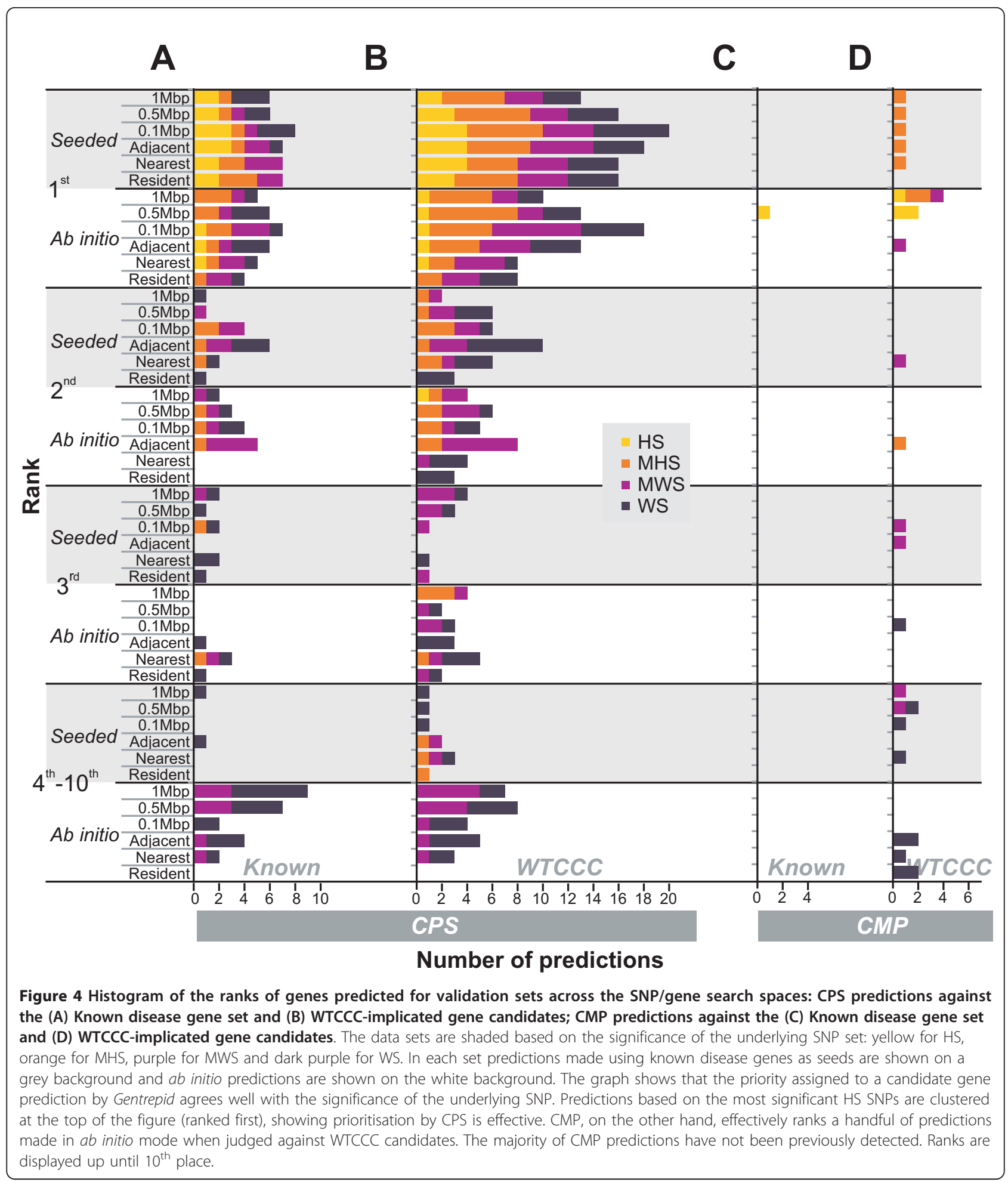

ranged between 0.35 to 0.50 , while CPS ab initio was between 0.40 and 0.67 . CPS had higher sensitivity in the $\mathrm{NN}$ gene selection sets compared to the BY sets. For CMP, the sensitivity was low due to few predictions. The specificity of the system ranged between 0.55 and 1 .
For CPS and CMP, specificity was less for the NN gene selection sets compared to the BY sets. For instance for the WS SNPs validated against known disease genes, CPS seeded had a specificity between 0.97 and 0.99 for the BY gene sets but only 0.92 to 0.97 for the NN gene 


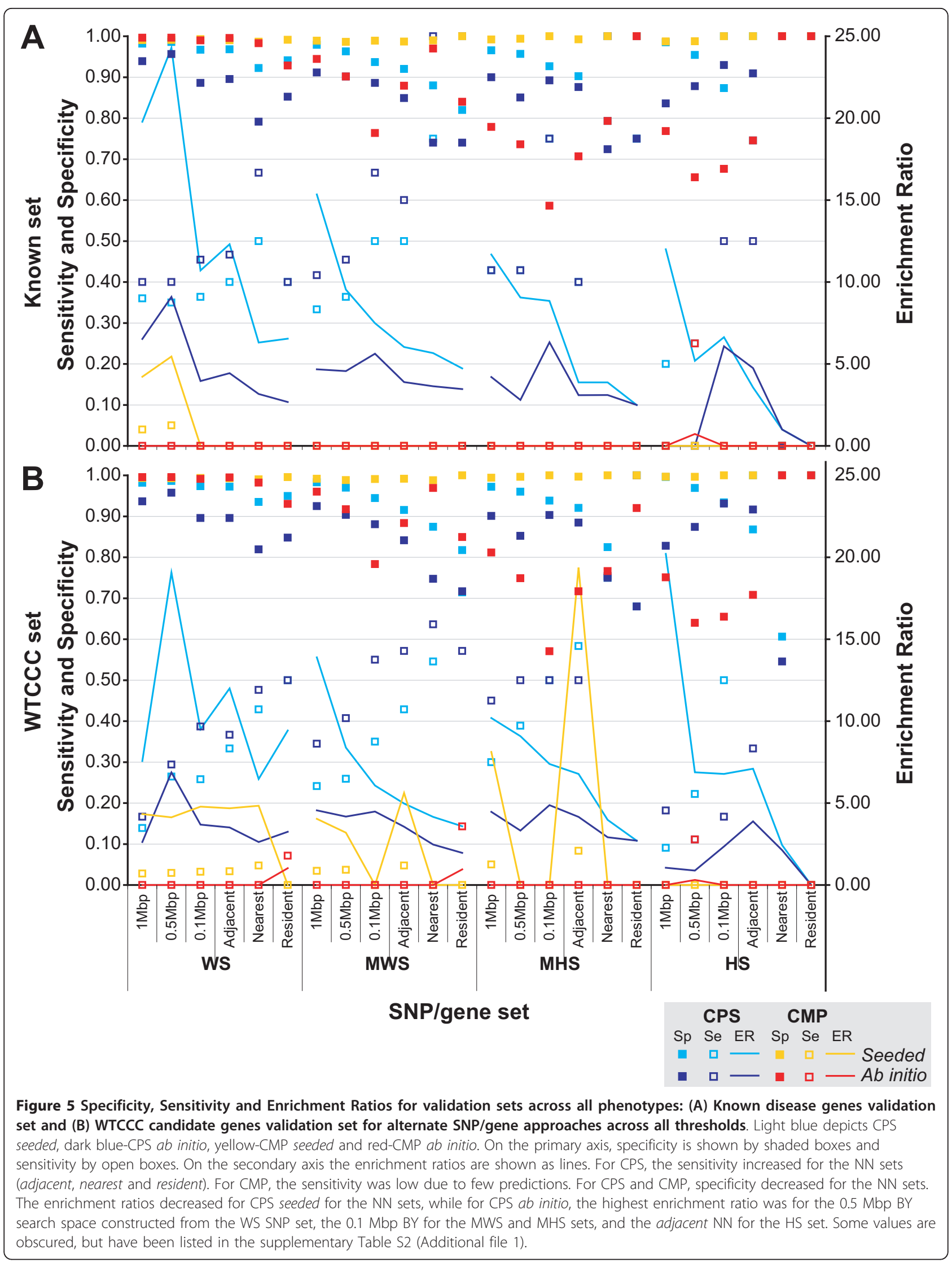


sets. The ER of the system components varied between 1 (no enrichment) and 24.29 fold enrichment. For CPS seeded ERs decreased with NN gene set size, while for CPS ab initio, the maximal ER was for the 0.5 Mbp BY gene set for the WS SNPs, but the smaller 0.1 Mbp BY gene set for the MWS, MHS and HS SNP sets. Similar results were obtained with the WTCCC validation set. CMP seeded and $a b$ initio benchmarked poorly against the known validation set, but CMP seeded performed much better on the WTCCC validation set. As for CPS, the largest ERs for CMP seeded were returned for the MWS and MHS data sets using the adjacent gene sets, and similar ERs for all the remaining approaches (except the resident) using the WS data set.

\section{Comparison to random controls}

As another test of the system, we compared predictions based on the WTCCC SNPs with predictions based on randomly generated SNP data. This test allows us to get a better handle on variables such as which gene selection sets are optimal, or if the signal-to-noise ratio begins to decrease as we decrease the significance level, as would be expected. We ran 1000 permutations of randomly selected SNPs for each disease, mode (seeded/ $a b$ initio) and module (CPS/CMP) set. As an indicator of performance, we calculated the log ratio of the number of predictions generated from the real data to the average from the randomly generated sets (Figure 6). A positive ratio indicates better performance on real versus random data, a neutral score no difference from random, and a negative ratio poorer performance. For example, if the WTCCC SNPs are really phenotype-specific at a particular significance level, we would expect a larger number of predictions by the real data than by random SNPs.

When predictions made in seeded mode using phenotype-related SNPs were compared to predictions based on random SNPs across the different gene selection sets, the performance of the system was mode and module specific. CPS seeded performed best on the NN sets. CMP seeded performed best on BY sets, but CMP $a b$ initio performed best on the NN sets. CPS ab initio was the worst performing module, mode combination. Seeded mode performs best across all SNP/gene approaches, whilst $a b$ initio performs the best for the $\mathrm{NN}$ sets. CPS performed the best for the NN sets, while CMP performs well across all SNP/gene approaches.

When predictions made in seeded mode using phenotype-related SNPs were compared to predictions based on random SNPs across the significance thresholds, CPS had a positive ratio for the MWS-, MHS- and HS-implicated loci. CPS $a b$ initio had a positive ratio for the MHS and HS sets. For CMP, both seeded and $a b$ initio modes generally had positive ratios for the MHS and
MWS set, neutral performance for the HS set, and a negative ratio for the WS set. In summary, the best performances were on the MHS and MWS thresholds across all the modules.

The poor performance of CPS on the WS sets in these tests using random data as compared to the benchmarks performed on previously discovered disease genes was surprising. Examining the random data shows that nonspecific pathways generated a disproportionate number of CPS predictions which led to an increased rate of false positives. An example is the cytokine-cytokine receptor pathway from KEGG which retrieves generic cytokines which are not cognate ligands for the retrieved cytokine receptors. Using the MHS data, the system performs better than random when pathway data is available because of the higher statistical significance of the SNPs in this dataset, but the number of loci is diminishing to the point where it is not possible for CPS to make a prediction. This is also a reflection of the dependence of the analyses on the quality of current databases and annotations.

The same significance thresholds used in CMP $a b$ initio across all the SNP/gene approaches do not take into account that the appropriate threshold may be search space dependent, as there are more genes in sets such as the $1 \mathrm{Mbp}$ compared to the adjacent. Post-filtering the candidate genes based on the number of times they appeared in the random simulations was one approach we took to reduce the number of false positives in the phenotype-specific predictions. Fewer predictions were filtered from the $\mathrm{NN}$ sets for all phenotypes, which indicates there was most likely less noise in these search spaces. The gene predictions in the more selective sets were robust when filtered against the random simulations, suggesting genuine predictions. As in the case of CPS, CMP $a b$ initio predictions require multiple loci which are fewer in the genetically more selective MHS and HS sets.

\section{Comparison to other systems}

Our system focuses on the use of the protein knowledge base for predictions of candidate disease genes from implicated regions but there are other tools and methods that utilise alternate annotation information to perform predictions. Previously, we compared Gentrepid to 6 other candidate gene prediction systems using linkage analysis data against GWA results for type II diabetes [17]. Here, we compared the performance of our system on the GWAS data to two other candidate gene prediction tools currently available online: GRAIL [36] and WebGestalt [37]. GRAIL [36] identifies relationships amongst genomic disease regions by text mining PubMed abstracts and assessing gene relatedness. WebGestalt [37] performs gene set enrichment analysis given 


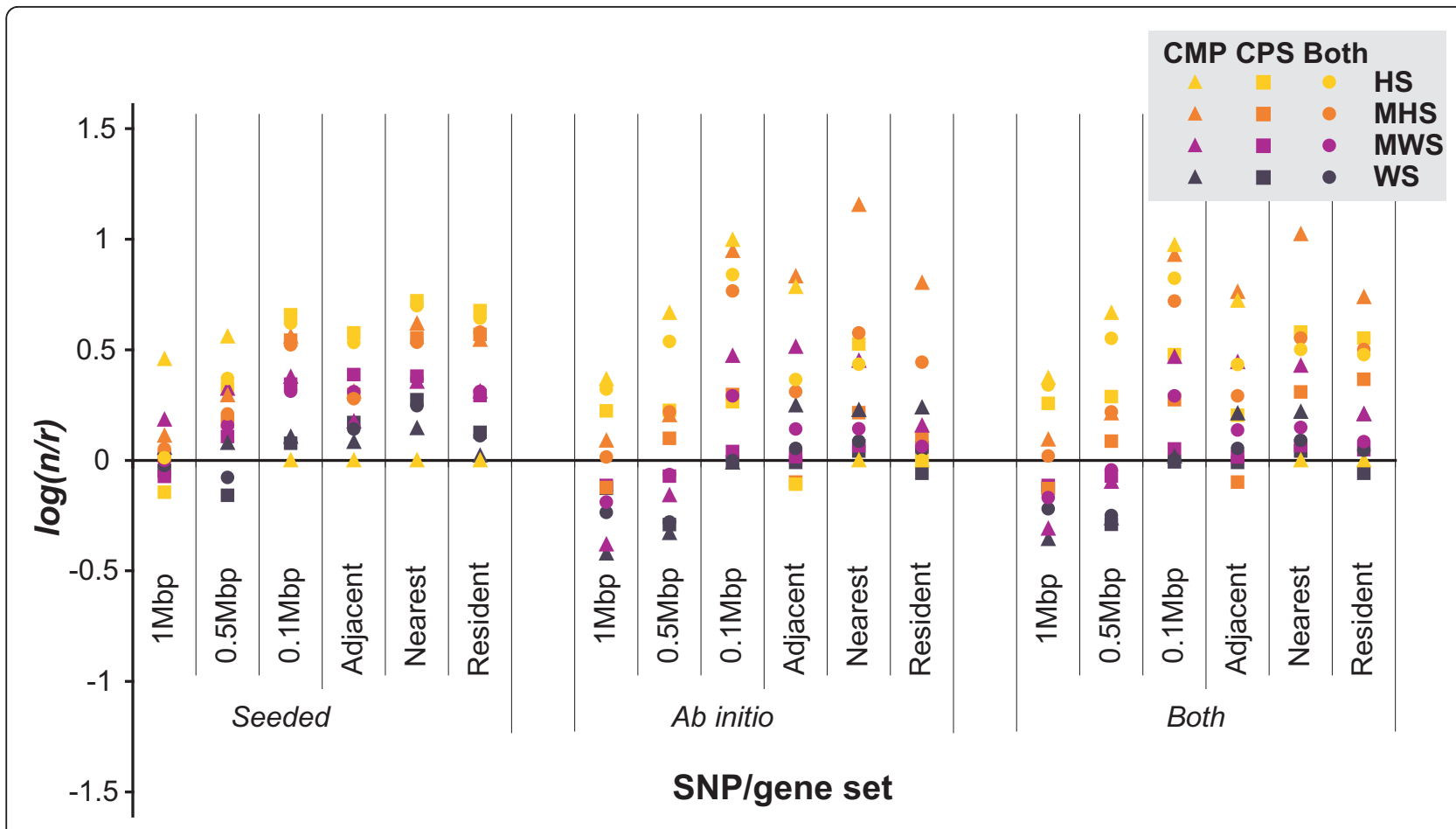

Figure 6 Performance of the different modules on the GWAS data compared to randomly generated data. Each point represents the log of the ratio of predictions for a phenotype-specific data set compared to a similar randomly generated SNP set. A positive ratio indicates better performance by the system. For the HS and MHS sets, the system performs better under most SNP/gene approaches, with the MHS set performing the best. In the MWS and WS sets, performance depends on the SNP/gene approach used to map the search spaces, with the NN gene set outperforming the BY sets. The nearest, adjacent and $0.1 \mathrm{Mbp}$ approaches have the best performance.

a list of genes or SNPs which it maps to genes using the array specific list of genes. We used the HS and MHS SNP sets to perform our analyses. Because GRAIL accepts a list of SNPs or disease regions and performs its own SNP-to-gene mapping based on LD, we used the gene set generated by GRAIL to perform the remainder of the analyses. Using LD to cluster the SNPs into distinct loci returned similar, but not always, identical results to the naive clustering method used in this study (Additional file 1, Table S5). For instance, the SNPs in the MHC locus are all in high LD over long stretches, yet the region is interrupted by multiple recombination hotspots [38]. As a result, the naïve clustering method returns multiple associated loci for the MHC region, while the LD method clumps all the SNPs into one large associated locus. The gene search spaces also differed between the LD method and the adjacent mapping implemented for the MHS and HS SNP sets. The average number of genes per locus varies for each disease, ranging from 1 to 27 genes per associated region (data not shown) with the LD approach. As described, the adjacent mapping would have at most 4 genes as candidates. Also, the adjacent mapping does not restrict the genes to be in LD with the associated locus so for some cases genes at greater distances from the associated region are analysed. An example is two Ephrin receptors (EFNB2 Ephrin-B2 and EPHA7 EPH receptor A7) which Gentrepid predicted as candidates for coronary artery disease in the adjacent gene set. Gentrepid made more predictions for the adjacent gene search space than it did for the LD search space for the HS SNP set, but made fewer predictions for the adjacent mapping in the MHS SNP set than from the LD (Additional file 1, Table S6). This is most likely due to a loss of power as the size of the gene set increases.

To compare the available web-based methods, we ran Gentrepid, GRAIL and WebGestalt on the HS and MHS sets for each disease using the LD mapped gene search spaces created by GRAIL. We considered a result to be a prediction if the statistical significance of the annotation returned was $p<0.05$. Overall, WebGestalt and GRAIL returned more candidate gene predictions and more regions with predictions than Gentrepid (Additional file 1, Table S7). But many of the predictions made by GRAIL and WebGestalt were genes from the same locus, indicating a higher false positive rate. We also calculated the sensitivity, specificity and enrichment ratio of the different methods using the WTCCC candidate genes as the validation set (Additional file 1, Table S8). Specificity was high for all the systems, so for more 
insight into performance we compared the sensitivity using the MHS set (Figure 7). GRAIL had the highest sensitivity with the PubMed abstract method, than when given seeded regions. Gentrepid CPS had similar sensitivities for both $a b$ initio and seeded modes, which remained the same when the $p$-value threshold was lowered $(p<0.01)$. Of note, the sensitivity values are much lower across all the methods using genes selected by linkage disequilibrium compared to the different mapping approaches considered in this study.

\section{Discussion}

GWAS are a valuable approach to identification of loci involved in disease phenotypes. In this work, we developed a method for analysing GWA data that uses a combined statistical and bioinformatic protocol to sort the genotype-phenotype signal from the noise. We adopted a double sift approach, based on genetic and biological knowledge, to identify likely causal genes in selected sets of phenotype-associated SNPs comprising up to $0.2 \%$ of genotyped SNPs. This method has revealed hidden information that was missed when the analysis relied solely on the SNPs of highest statistical significance. This may explain some of the missing heritability in GWAS [9]. The biological information employed utilizes pathways and domain-based similarity to find relationships between multiple genes associated with genetic data for specific phenotypes.

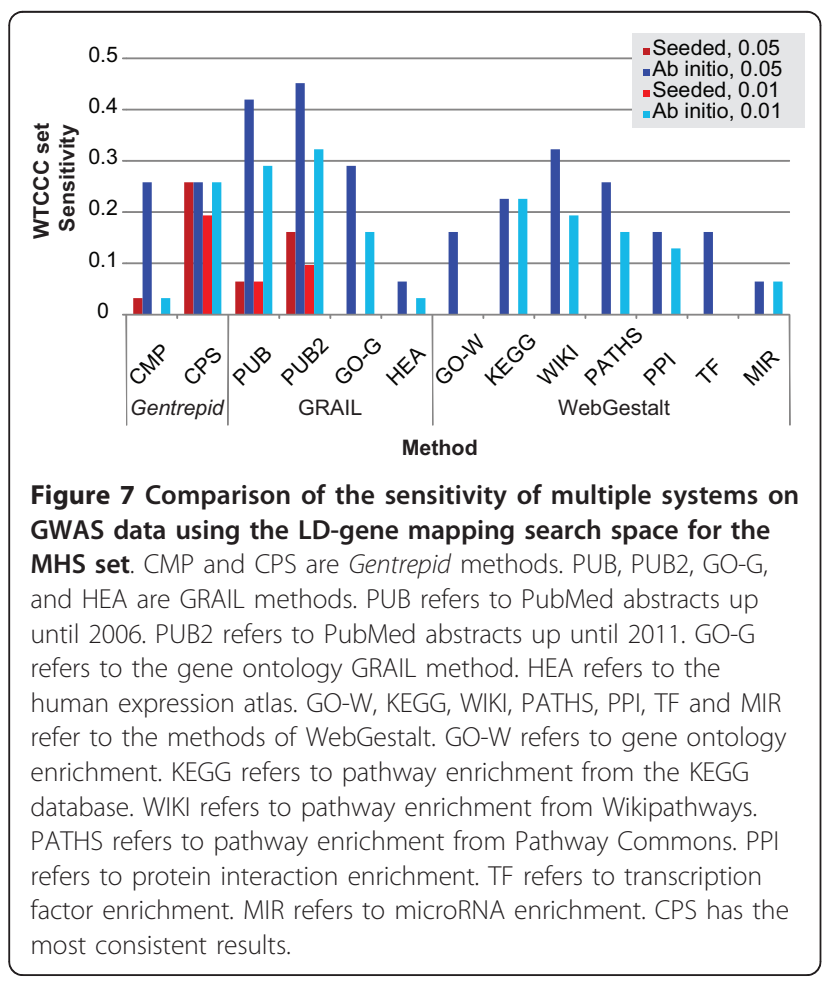

\section{Value of systematic SNP significance investigation}

The lower statistical thresholds used in the study capture a large number of phenotype-SNP associations that were not revealed in previous studies. Although lowering the statistical threshold may increase the number of false positive SNPs reported, regions that were otherwise missed by the high significance threshold cut-off adopted in the original study clearly contain phenotypespecific information that departs from random data. The lower statistical significance of these associations may reflect either the rarity of the alleles or those with small effect sizes. Both these types of alleles are believed to be major contributors to the disease phenotypes as the majority of common SNPs associated with the phenotypes studied fail to explain the heritability of many diseased individuals [9]. The replication of known loci in the data of lower significance supports the value of this approach.

\section{Selecting gene sets associated with each SNP set}

Some studies have suggested $[10,11]$, for example those on long range regulation of genes, that the location of controlling elements may be distal to the actual transcripts and protein-coding regions themselves. For this reason, gene sets were selected around SNPs in six different ways to investigate how these SNP to gene selection assumptions affected predictions. The different assumptions, such as the bystander approach, increase the study's gene coverage of the genome, potentially capturing longer range associations between SNPs and genes. Whether these longer range associations are regulatory or arise from linkage disequilibrium remains an open question.

An unavoidable accompaniment of using distancebased gene selection approaches is the introduction of noise into the results which therefore requires stricter filtering. A single associated locus captures a set of at most 4 genes in the proximity-based $\mathrm{NN}$ approaches, but in the distance-based BY approaches, some loci that are in gene dense regions link to many more genes. In the largest gene sets tested, on average, there were 16 genes to a $1 \mathrm{Mbp}$ locus. Many of the predictions made by Gentrepid are for the largest loci: $1 \mathrm{Mbp}$ BY, 0.5 Mbp BY and adjacent NN. In many instances the predicted gene is not the nearest gene to the implicated SNP (Table 2). This may truly demonstrate long range regulatory effects, or alternatively the inclusion of more genes may simply increase the chances of predictions. The most successful approaches, as judged by the enrichment ratios, specificity and sensitivity measurements on the validation sets, are the adjacent and 0.1 Mbp approaches. Both these sets have similar search space sizes and contain less noise than the larger BY sets. However, there may be an element of self-selection 
in this result, if geneticists typically only scan the immediate vicinity of the locus studied for the disease gene. Even so, the more generous SNP/gene assumptions did not unduly lower the performance of the system, with multiple instances of consensus amongst predictions across the different sets [39]. Enrichment ratios varied from 15 to 25 depending on the thresholds. For the maximal search space of 3000 genes, this is roughly equivalent to $120-200$ disease candidates per phenotype: a number that is feasible to scan with current sequencing technologies.

\section{Performance in seeded versus $a b$ initio mode}

Predictions based on known disease genes are inherently limited by what is already known about a phenotype. In seeded mode, Gentrepid is an effective tool to assist in the discovery of phenotype-related genes in novel loci. The $a b$ initio methodology is a powerful discovery tool for finding novel genotype-phenotype relationships for complex diseases. For diseases with Mendelian inheritance, ab initio mode is also likely to be advantageous if only a small percentage of cases arise from known disease genes.

CPS seeded mode is generally a more powerful discovery tool when retrieving novel genes associated with pathways involving disease genes previously linked to the phenotype. In this mode, the candidate gene search space is enriched for known disease pathways, increasing the chances of retrieving genes that share this pathway. In contrast, $a b$ initio mode only considers genes within the candidate loci and excludes many of the confirmed disease genes: a more agnostic approach which may be informative.

In addition to the constraints described above for $a b$ initio predictions, the success of Gentrepid predictions using known disease genes depends on how informative these genes are for the phenotype. A number of factors influence the system's ability to make predictions including the quality of the input GWA data for the specific phenotype, and genome coverage of pathways specific to phenotype. Even when the entire genome is considered, only $57 \%$ of characterized genes have Gentrepid annotations and are thus potentially predictable as candidates. Most of this coverage is due to Pfam domains, while pathways cover up to $20 \%$ of annotated genes (Figure 2). Thus the system is unable to make predictions for around $40 \%$ of the genome which may or may not be associated with the phenotype. Outside these phenotype-specific constraints, a threshold number of loci are required for the prediction to be significant. As discussed, generic pathways that are not highly informative or specific, increase the chances of random predictions by CPS, as do common domains by CMP (e. g. Ras PF00071).

\section{Performance of CMP versus CPS}

In our previous benchmark, which used a dataset of Mendelian diseases developed by Turner et al [40], we found CPS more effective in retrieving candidates. Using GWA data for complex diseases, the domain-based CMP module of Gentrepid made many plausible predictions. The auto-detected domain comparison in CMP removes the need to rely on the current annotations of human proteins, which are still lacking [41]; or on whole gene sequence-similarity which is less accurate [42]. However it was interesting that CPS was still more effective in replicating known disease genes including some loci where a disease gene has not previously been allocated. Of the 29 known disease genes, 16 were predicted by CPS. The predictions made by CMP may be spurious, although the random simulations suggest otherwise. Alternatively, the superior performance of CPS in replicating known disease genes may be a selection effect. The genes within the sets were determined to be disease causing based on known disease pathways and interactions. Specifically, geneticists may have been searching for disease genes for complex diseases based on experience gained from Mendelian diseases. In the case of Mendelian diseases, this approach may apply, as penetrance is high for monogenic disorders. Overlapping functionality arising from similar domain structures would not be a very strong predictor for Mendelian diseases, as genes with similar functions would not have highly penetrant phenotypes. Genes with overlapping functions may mask each other's defects, be insufficient to cause the disease alone, and perhaps only fractionally increase the risk. In complex or polygenic disorders, genes with overlapping functions that are mutated or dysregulated may be more common, and hence predictions by CMP may be more suited for gene discovery in these disease states.

\section{Performance on validation sets}

Gentrepid was capable of replicating genes already implicated by past genetic studies and the WTCCC GWAS. For loci flagged by the GWA study that were previously noted in OMIM, CPS successfully prioritised the known disease genes. For the genes determined by the WTCCC as likely candidates, either CPS or CMP was capable of predicting the candidates. As the two sets were generated from different genetic sources, it is not unusual for the system to perform differently on both. The known disease genes were determined through family linkage analysis studies, but the WTCCC gene validation set was generated from SNPs that are population based. It may be that the known disease genes are family specific or "private" and were not in the population studied by the WTCCC. The WTCCC candidates were selected by looking at the nearest 
genes, and not further, possibly missing other real candidates. Therefore differentiating false positives from true positives is almost subjective.

Although the data were averaged across the seven WTCCC phenotypes for this analysis, the performance of the system is somewhat phenotype dependent. The stringency threshold on some phenotypes has to be lower to compensate for genetic heterogeneity in diseases such as hypertension. For the autoimmune diseases where highly significant results are within gene dense regions such as the MHC locus or the cytokine cluster on human chromosome 5, the identification of the causal gene cannot be resolved through data mining analyses as all genes in the region share similar functions and protein structures. However the system does give some important information in these instances by identifying the domain or pathway that is being represented by the gene clusters, which in the previous example are genes regulating immunity. It is also important therefore to use all the genes within the cluster for analysis, and hence the SNP/gene distance based approach, as less common pathways within the cluster may be important in the multiple loci analyses that the system performs. The genetic and biological complexity of the diseases is demonstrated in the raw data from the GWA SNP analysis. To decipher this complexity, the biomolecular and protein analysis automatically detects commonality between multiple loci detected and thus, to some extent, compensates for current statistical genetic methods used on GWA data that test each $\mathrm{SNP}$ is isolation from the others.

\section{Comparison to other systems}

Each method studied here has its strengths and weaknesses which should be kept in mind during use. For instance, WebGestalt [37] looks for gene annotation enrichment but does not take into account gene duplication found within the same locus. Gene clusters of similar genes such as those in the MHC locus are given equal weight as those from multiple regions and would inflate particular results. GRAIL [36] and Gentrepid on the other hand, correct for this by adjusting the calculations so genes from the same associated region are not counted multiple times. Many of the GRAIL predictions were made through the text of recent PubMed abstracts, while fewer significant predictions were made with the text prior to GWAS publications, indicating that the results are mostly returning what we know and few de novo candidates. In short, GRAIL is acting as a retrieval tool. Gentrepid appears to be predicting novel candidates with its functional domain-based approach. Also, very few of the predictions overlapped between the different data sources used in each system. This emphasizes that one source of data may not be sufficient to make candidate gene predictions and that using alternate tools and data is wise, although these need to be carefully tested and understood in isolation.

Several advantages of Gentrepid are: it allows analysis of large datasets such as the MWS and WS set used in this study; like GRAIL, Gentrepid allows users the flexibility to enter genes or genome intervals, but uses different data sources for predictions. Gentrepid performs similar enrichment analysis to WebGestalt but accounts for multiple genes implicated from the same region.

\section{Conclusions}

In conclusion, we performed an extensive analysis of the Gentrepid system using GWA data. The approach used four sets of significant SNPs at different significance thresholds. SNPs were mapped to the genome in six different ways and the resulting search spaces analysed with the Gentrepid candidate gene prediction system. The results show that using what is known about the disease (seeded) as well as a blind approach (ab initio) is beneficial in the discovery and prediction of candidate disease genes. Further to this, using a less stringent SNP association threshold allows true signals to be detected which can be filtered using biomolecular information. Also, when using gene selection approaches which include genes that are not the nearest gene to the implicated SNP, Gentrepid makes significant predictions without unduly lowering the performance of the system. As the predictions remain dependent on what we already know in the protein knowledge base and on disease information, the system is only as good as the underlying databases. Further detailed work on discovery and annotation is required to take advantage of the existing GWA data. We believe this method to be an important tool in analysing GWAS as current methods are less flexible and require more data processing.

\section{Methods}

\section{WTCCC data}

We obtained SNP data from the WTCCC [33] case-control studies of seven diseases: bipolar disorder (BD), coronary artery disease (CAD), Crohn's disease (CD), hypertension (HT), rheumatoid arthritis (RA), type I diabetes (T1D) and type II diabetes (T2D). The WTCCC GWAS used the Affymetrix chip set with approximately 500,000 known SNPs (Affy500k), with SNP positions referenced to the human genome sequence assembly from NCBI (build 35). We mapped these SNPs to 489,763 autosomal SNPs on the genome assembly (build 36.3 ), and 459,231 SNPs following WTCCC quality control [33].

\section{OMIM known disease genes}

We extracted known disease genes and loci from the OMIM database [35] Morbid Map flat file by 
performing a text search for the disease name or parts thereof. These were then manually filtered for relevant loci.

\section{Choice of SNP significance thresholds}

An initial set of associated SNPs was filtered from the summary data of SNPTEST [43], a program that performs a series of association tests on the genotypes obtained from the case-control studies. The $p$-value of the trend test statistic (Cochran-Armitage test) [44] of the additive genetic model was used as a test statistic for SNP significance. The levels of significance chosen as the SNP association thresholds were determined using quantile-quantile (Q-Q) plots of the datasets (Additional file 2, Figure S1). The Q-Q plots were constructed by plotting the observed $-\log _{10}\left(p_{G W A}\right)$ of the SNPs against the expected $-\log _{10}\left(p_{G W A}\right)$, constructed under the null hypothesis that there is no association between the SNPs and the phenotype. Visual inspection of the Q-Q plots shows the distribution of test statistics of the observed SNPs for each phenotype begins to deviate from the expected distribution under the null hypothesis near $p_{G W A} \approx 10^{-2}$ and is distinctly different at $p_{\text {GWA }} \leq 10^{-3}$.

Four different $p$-value thresholds were used to create four associated SNP data sets for each phenotype: a weakly significant set (WS, $p_{G W A} \leq 10^{-3}$ ), a moderatelyweak significant set (MWS, $p_{G W A} \leq 10^{-4}$ ), a moderatelyhigh significant set (MHS, $P_{G W A} \leq 10^{-5}$ ), and a highly significant SNP set (HS, $p_{G W A}<5 \times 10^{-7}$ ). The final HS set is equivalent to the threshold used in the WTCCC study, where the $p$-value was determined based on the $a$ priori probability of association, and not the typical multiple-comparison or Bonferroni correction [33].

SNPs within the sets were clustered based on physical distance to one another through a naïve distance-based clustering process: a SNP within $50 \mathrm{Kbp}$ of another SNP was considered to form a cluster. This value was chosen based on the average size of haplotype blocks [45].

\section{Construction of candidate gene search spaces}

We used Gentrepid to predict and prioritise candidate disease genes selected from phenotype-associated gene sets generated from the SNP loci. Gene sets were constructed using one of two major assumptions: diseaseassociated SNPs are either resident in, or adjacent to, the disease gene; or the disease-associated SNPs may be near, but not closest or adjacent to, the disease gene. The first assumption we termed the nearest neighbour gene selection approach $(\mathrm{NN})$ and the second assumption the bystander approach (BY).

For the NN approach, three sets of genes were created: a set containing genes with SNPs internal to the gene boundary defined by RefSeq [26], termed the resident set; a second set with SNPs resident in the gene or directly adjacent to it, termed the nearest set; and a third set with the SNPs either resident in, or directly adjacent to, the four nearest genes, termed the adjacent set. The nearest set corresponds to the set commonly selected by nearest neighbour approaches in most recent GWAS [3]. In the adjacent set, genes on both strands of the chromosome were considered in both the 5' and 3' direction. For both the nearest and adjacent sets the physical distance between the SNP and the gene was not used as a constraint.

For the BY approach, three intervals of different sizes were tested. These values were chosen based on average distances of transcriptional regulatory elements from the genes they control $[46,47]$. Genes on both strands around each of the SNPs were pooled from flanking intervals of $0.1 \mathrm{Mbp}, 0.5 \mathrm{Mbp}$ or $1 \mathrm{Mbp}$ in width.

SNP and gene density are non-uniform across the genome and gene sizes vary, all of which influence the number of positional gene candidates available for analysis. To test for bias due to SNP coverage by the Affymetrix chip set, we first checked the SNP distribution across the genome. SNP positions and the frequency of SNPs in different gene regions (exonic, intronic, UTRs) and intergenic locations of the genome were calculated by creating density plots. To determine if gene coverage was affected by the various SNP-to-gene search space construction assumptions, we calculated the percentage of genes in the genome covered by SNPs on the Affy500K chip set using each approach. We also wished to determine if these genes were represented in Gentrepid by associated pathways and domains.

\section{Prediction and prioritisation of candidate genes}

To determine which SNPs are more likely to contribute to the disease phenotype, a set of analyses were performed using direct SQL queries of the in-house Gentrepid database https://www.gentrepid.org. Gentrepid's two modes of input, seeded mode and ab initio mode were used to determine the common properties of phenotype-specific genes. Seeded mode is assisted by phenotype-associated genes from OMIM as seeds (Additional file 1, Table S1). Ab initio mode uses only genes pooled from the search spaces.

Genes in each data set were prioritised based on phenotype-associated common pathways (via CPS) and common domains (via CMP). In previous work using CPS, pathways containing at least two genes from distinct loci were ranked based on the total number of loci involved, as described in George et al [16]. This is not entirely satisfactory because it favours large pathways. For instance, a pathway containing a large number of genes may be selected over a more pertinent smaller pathway or a subnet. To test the likelihood of a pathway 
being associated with a phenotype, genes were bi-partitioned based on whether they were associated with (a) the phenotype in question and (b) the pathway in question. To calculate the significance, Fisher's exact test was performed using the fisher.test function in R http:// www.r-project.org/[48]. For CPS seeded and ab initio, gene predictions were filtered based on the statistical significance of the pathway using a threshold of $p_{\text {path }}<$ 0.05 , and prioritised based on the lowest $p$-value score of the pathway they shared.

For CMP, the domains of phenotype-specific genes were queried from the database and compared to domains of other phenotype-specific genes in the data set ( $a b$ initio) or domains of known disease genes (seeded), as described in George et al [16]. For CMP seeded, predictions are based on a pair wise similarity score between the candidate and a known disease gene between 0 (no similarity) and 1 (identical) [16]. Using a benchmark set of oligogenic diseases with Mendelian inheritance suggested by Turner et al [40], we previously determined that a pair wise similarity score of 0.4 between the test gene and the known disease gene is a conservative threshold above which the test gene can be considered a candidate [16]. Results above the threshold score of 0.4 were filtered and prioritised. In CMP $a b$ initio mode, the domain combination was tested for over-representation in the constructed intervals compared to the genome as a whole through upper and lower significance tests, based on a range of expected values relating to domain correlation within genes. The expected number of domains was calculated based on the value of $p$, representing the extrema of the level of correlation between domains in genes $\left(p_{\text {min }}\right.$, $\left.p_{\text {max }}\right)$, and more specifically the likelihood of occurrence of the domain combination by chance [16]. Within a gene, domain duplications are reasonably common [25] leading to an anomalously low $p_{\max }$. Thus a revised $p_{\max }$ which ignores multiple copies of domains was calculated to correct for this effect $\left(p_{\text {max_unique }}\right)$. The gene predictions were filtered on significance based on the three $\chi^{2}$ tests $\left(\chi_{\text {min }}^{2}, \chi_{\text {max_unique }}^{2}\right.$ and $\chi_{\text {max }}^{2}$. A $\chi^{2}$ value greater than 7.88 is significant at the 0.005 level, but we adopted more conservative values of $\chi_{\text {max_unique }}^{2} \geq 10^{5}$ for multidomain proteins, and $\chi^{2 \min } \geq 10^{2}$ for single domain proteins. The predictions were then filtered against random simulations described below to remove false positives. Finally, the three $\chi^{2}$ scores were correlated with the random predictions using the Spearman's rank correlation coefficient test to choose the best metric for prioritisation. Based on this test $\chi^{2 \text { min }}$ was chosen.

As a control we selected random SNP sets of similar size to the phenotype-specific data and mapped these to genes using the same protocols described above $(\mathrm{NN}$,
BY). The number of SNPs selected for each search space was similar to the number of clusters formed by the SNPs in the WTCCC data, and not the exact number of significant SNPs. This was done to account for clusters in the phenotype-specific data due to linkage disequilibrium or SNP-disease association. For each random set, we ran Gentrepid CPS and CMP in both seeded and ab initio modes, and tracked predictions and significance scores. The randomization results were averaged across 1000 replications.

\section{Validation of predictions and metrics}

We took several approaches to assess the ability of the two Gentrepid modules to extract positional candidates. Firstly, we studied the ability of Gentrepid to extract and prioritise known disease genes and loci from the phenotype data. When known disease genes were employed as seeds for Gentrepid predictions, a leaveone-out cross validation technique was used. In this process, known disease genes were iteratively removed as seeds during the prediction process, and the resulting rank of the withheld gene was then assessed. The ranks of known gene predictions in $a b$ initio mode were also calculated. Secondly, we assessed the Gentrepid results against genes associated with the HS SNPs by the WTCCC. Finally, predictions on the GWA-implicated loci were compared to predictions made by Gentrepid on the random data for the chosen levels of stringency using both the NN and BY gene selection assumptions. We calculated the specificity, sensitivity and enrichment ratios for each of the validation sets as described in our previous work [17] and also plotted ROC curves for an overall comparison of the system (Additional file 3, Figure S2).

\section{Comparison to other online methods}

We selected GRAIL [36] and WebGestalt [37] as online tools for comparison because they both take input from GWAS data, and perform gene annotation enrichment analysis similar to Gentrepid. GRAIL makes three independent predictions using three different sources of data: text mining of PubMed abstracts, Gene Ontology (GO) annotations [49] or mRNA expression levels from The Novartis Gene Expression Atlas [50]. Genes are given a significance score [36]. WebGestalt performs gene enrichment analysis on a user-defined gene/protein list or a list of SNPs from typical GWAS arrays such as those from Affymetrix or Illumina. The analysis is performed by searching for enrichment of gene annotations from GO, pathways from KEGG, Wikipathways and Pathway commons, transcription factor binding site motifs and microRNA target enrichment. Genes are not individually ranked, but a $p$-value is calculated for every annotation returned as a result [37]. 
To use a standard set of genes across all three tools, we had to utilize the GRAIL gene search space as it was the most restrictive. The GRAIL gene search space is generated automatically from a user-defined list of SNPs according to the linkage disequilibrium of the associated locus. For each disease, we uploaded the list of associated significant SNPs for the HS and MHS set. GRAIL performs LD mapping and returns a list of query genes. This LD gene list was then used as input for both Gentrepid and WebGestalt.

We ran GRAIL using the PubMed Text 2006 and 2011, GO 2006 and Novartis databases. A seeded search can also be performed in GRAIL by including genomic regions known to be associated with the disease. We used the OMIM set defined earlier as the known gene set and ran queries on the same databases. For each gene in the search space, we stored the prediction $p$ values for each data source.

We ran WebGestalt enrichment analysis on the same gene lists analyzed by GRAIL. We used the "hspaiens_genome" as the reference gene list for all enrichment analyses and ran the web tool using the default settings, except for the significance levels which were set to $p<$ 0.05 (default of Top 10). We performed GO analysis, KEGG analysis, Wikipathways analysis, Pathway Commons analysis, transcription factor target analysis, microRNA target analysis, and protein interaction network module analysis. For each gene, we stored the most significant $p$-value for each analysis. We considered two sets of predictions using thresholds significance $p$-values of either 0.05 or 0.01 .

We ran Gentrepid on the LD-mapped gene search space, using both CPS and CMP in seeded and ab initio mode. We stored the CPS $p$-values as the prediction $p$ values. CMP scores were evaluated at scores of either 0.4 or 0.8 for seeded CMP, and $\chi^{2 \text { min }}$ scores of either 100 or $10^{5}$ for ab initio CMP.

We then compared the $p$-values of the genes across all the systems. We also calculated the specificity, sensitivity and enrichment ratios on the WTCCC validation set and compared across the different methods.

\section{Additional material}

Additional file 1: Gentrepid validation gene sets and additional benchmarking results.Table S1 OMIM phenotype associated genes used as seeds for the seeded mode and as the known disease gene validation set.Table S2 Genes included in the known validation set. Table S3 Genes included in the WTCCC validation set. Table S4 Specificity, Sensitivity and Enrichment ratios for validation sets across all phenotypes. Table S5 LD versus naïve clustering. Table S6 Comparison of the number of significant Gentrepid predictions between LD and adjacent gene selection sets. Table S7 Total numbers of significant predictions across Gentrepid, GRAIL and WebGestalt. Table S8 Specificity, Sensitivity and Enrichment ratios for WTCCC validation set for Gentrepid, GRAIL and WebGestalt.
Additional file 2: Figure S1 Q-Q plots of expected values of the associated trend test $p$-values versus observed generated for each phenotype in black and uniform distribution in grey.

Additional file 3: Figure S2 ROC curves for Gentrepid on known and WTCCC validation sets. CPS is represented by the dashed lines, CMP by the filled lines. The colors indicate the SNP-to-gene mapping set used. The first column from the left are the results for the known validation set using seeded mode, The second column are the known validation set under $a b$ initio. The third column is the WTCCC validation set seeded results. And the fourth column the WTCCC set, ab initio. The top panels are the HS sets. The next set of panels the MHS set, the third MWS and the bottom panels the WS set. The grey line in each plot represents what a random guess should give. CPS is above the line for most cases. CMP is below. CPS with the $0.1 \mathrm{Mbp}$ or adjacent set performs the best.

\section{List of abbreviations}

GWAS: Genome-wide association studies; WTCCC: Wellcome Trust CaseControl Consortium; CPS: common pathway scanning; CMP: common module profiling; BD: Bipolar disorder; CAD: Coronary artery disease; CD: Crohn's disease; HT: Hypertension; RA: Rheumatoid arthritis; T1D: Type I diabetes; T2D: Type II diabetes; NN: Nearest neighbour approach; BY: Bystander approach; WS: Weakly significant set; MWS: Moderately-weak significant set; MHS: moderately-high significant set; HS: highly significant set.

\section{Acknowledgements}

This work was supported by the St. Vincent's Clinic Foundation [to M.A.W.] an NHMRC R. D. Wright Fellowship [to M. B.]; the Australian National Health and Medical Research Council [grant number 635512 to M.A.W., M.O. and D. F.]; the Ronald Geoffrey Arnott Fund [to M.A.W.]; and an Australian

Postgraduate Award through the University of New South Wales [to S.B.]. This study makes use of data generated by the Wellcome Trust Case-Control Consortium. A full list of the investigators who contributed to the generation of the data is available from the http://www.wtccc.org.uk. Funding for the project was provided by the Wellcome Trust [grant numbers 076113, 08475].

\section{Author details}

${ }^{1}$ Structural and Computational Biology Division, Victor Chang Cardiac Research Institute, Darlinghurst, NSW, 2010, Australia. ${ }^{2}$ School of Computer Science and Engineering, University of New South Wales, Kensington, NSW, 2052, Australia. ${ }^{3}$ Centre for Molecular and Biomolecular Informatics, Radboud University Nijmegen Medical Centre, Nijmegen, The Netherlands. ${ }^{4}$ School of Medical Sciences, University of New South Wales, Kensington, NSW, 2052, Australia. ${ }^{5}$ Molecular Cardiology and Biophysics Division, Victor Chang Cardiac Research Institute, Darlinghurst, NSW, 2010, Australia. ${ }^{6}$ Bioinformatics Division, The Walter and Eliza Hall Institute of Medical Research, Parkville, VIC, 3052, Australia. ${ }^{7}$ School of Life and Environmental Sciences, Deakin University, Geelong, VIC, 3217, Australia.

\section{Authors' contributions}

SB carried out the data mining and analysis, and worked on the design of the project and algorithms. $J L$ worked on the algorithms. MO worked on the CMP predictions. BG, DF and MB participated in the design of the study. MAW conceived the study, participated in its design, helped draft the manuscript and reviewed the results from the data analysis. All authors read and approved the final manuscript.

\section{Competing interests}

One of the authors (MAW) has a US patent application on the CMP methodology.

Received: 30 March 2011 Accepted: 13 November 2011 Published: 13 November 2011

\section{References}

1. Murcray CE, Lewinger JP, Gauderman WJ: Gene-environment interaction in genome-wide association studies. Am J Epidemiol 2009, 169(2):219-226. 
2. Risch $N$, Merikangas $K$ : The future of genetic studies of complex human diseases. Science 1996, 273(5281):1516-1517.

3. Wang K, Li M, Bucan M: Pathway-based approaches for analysis of genomewide association studies. Am J Hum Genet 2007, 81(6):1278-1283.

4. Zeggini E, Weedon MN, Lindgren CM, Frayling TM, Elliott KS, Lango H, Timpson NJ, Perry JRB, Rayner NW, Freathy RM, Barrett JC, Shields B, Morris AP, Ellard S, Groves CJ, Harries LW, Marchini JL, Owen KR, Knight B, Cardon LR, Walker M, Hitman GA, Morris AD, Doney ASF, The Wellcome Trust Case Control C, McCarthy MI, Hattersley AT: Replication of genomewide association signals in UK samples reveals risk loci for type 2 diabetes. Science 2007, 316(5829):1336-1341.

5. Scott L, Mohlke KL, Bonnycastle LL, Willer CJ, Li Y, Duren WL, Erdos MR, Stringham HM, Chines PS, Jackson AU, Prokunina-Olsson L, Ding C-J, Swift AJ, Narisu N, Hu T, Pruim R, Xiao R, Li X-Y, Conneely KN, Riebow NL, Sprau AG, Tong M, White PP, Hetrick KN, Barnhart MW, Bark CW, Goldstein JL, Watkins L, Xiang F, Saramies J, et al: A genome-wide association study of type 2 diabetes in Finns detects multiple susceptibility variants. Science 2007, 316(5829):1341-1345.

6. Saxena R, Voight BF, Lyssenko V, Burtt NP, de Bakker PIW, Chen H, Roix JJ, Kathiresan S, Hirschhorn JN, Daly MJ, Hughes TE, Groop L, Altshuler D, Almgren P, Florez JC, Meyer J, Ardlie K, Bengtsson Bostrom K, Isomaa B, Lettre G, Lindblad U, Lyon HN, Melander O, Newton-Cheh C, Nilsson P, Orho-Melander M, Rastam L, Speliotes EK, Taskinen M-R, Tuomi T, et al: Genome-wide association analysis identifies loci for type 2 diabetes and triglyceride levels. Science 2007, 316(5829):1331-1336.

7. Pearson TA, Manolio TA: How to interpret a genome-wide association study. JAMA 2008, 299(11):1335-1344.

8. Hristovski D, Peterlin B, Mitchell JA, Humphrey SM: Using literature-based discovery to identify disease candidate genes. Int J Med Inf 2005, 74(24):289-298.

9. Manolio TA, Collins FS, Cox NJ, Goldstein DB, Hindorff LA, Hunter DJ, McCarthy MI, Ramos EM, Cardon LR, Chakravarti A, Cho JH, Guttmacher AE, Kong A, Kruglyak L, Mardis E, Rotimi CN, Slatkin M, Valle D, Whittemore AS, Boehnke M, Clark AG, Eichler EE, Gibson G, Haines JL, Mackay TFC, McCarroll SA, Visscher PM: Finding the missing heritability of complex diseases. Nature 2009, 461(7265):747-753.

10. Kikuta $H$, Laplante $M$, Navratilova $P$, Komisarczuk $A Z$, Engstrom PG, Fredman D, Akalin A, Caccamo M, Sealy I, Howe K, Ghislain J, Pezeron G, Mourrain P, Ellingsen S, Oates AC, Thisse C, Thisse B, Foucher I, Adolf B, Geling A, Lenhard B, Becker TS: Genomic regulatory blocks encompass multiple neighboring genes and maintain conserved synteny in vertebrates. Genome Res 2007, 17(5):545-555.

11. Komisarczuk AZ, Kawakami K, Becker TS: Cis-regulation and chromosomal rearrangement of the FGF8 locus after the teleost/tetrapod split. Dev Biol 2009, 336(2):301-312

12. Roeder K, Bacanu S-A, Wasserman L, Devlin B: Using linkage genome scans to improve power of association in genome scans. Am J Hum Genet 2006, 78(2):243-252.

13. Jallow M, Teo Y, Small K, Rockett K, Deloukas P, Clark T, Kivinen K, Bojang K, Conway D, Pinder M: Genome-wide and fine-resolution association analysis of malaria in West Africa. Nat Genet 2009, 41(6):657-665.

14. Wacholder S, Chanock S, Garcia-Closas M, El ghormli L, Rothman N: Assessing the probability that a positive report is false: An approach for molecular epidemiology studies. J Natl Cancer Inst 2004, 96(6):434-442.

15. Pe'er I, de Bakker PIW, Maller J, Yelensky R, Altshuler D, Daly MJ: Evaluating and improving power in whole-genome association studies using fixed marker sets. Nat Genet 2006, 38(6):663-667.

16. George RA, Liu JY, Feng LL, Bryson-Richardson RJ, Fatkin D, Wouters MA: Analysis of protein sequence and interaction data for candidate disease gene prediction. Nucleic Acids Res 2006, 34(19):e130.

17. Teber E, Liu J, Ballouz S, Fatkin D, Wouters M: Comparison of automated candidate gene prediction systems using genes implicated in type 2 diabetes by genome-wide association studies. BMC Bioinformatics 2009, 10(Suppl 1):S69.

18. Badano $\mathrm{J}$, Katsanis $\mathrm{N}$ : Beyond Mendel: An evolving view of human genetic disease transmission. Nat Rev Genet 2002, 3(10):779-789.

19. Oti M, Snel B, Huynen MA, Brunner HG: Predicting disease genes using protein-protein interactions. J Med Genet 2006, 43(8):691-698.

20. Franke $L$, van Bakel $H$, Fokkens $L$, de Jong ED, Egmont-Petersen $M$, Wijmenga C: Reconstruction of a functional human gene network, with an application for prioritizing positional candidate genes. Am J Hum Genet 2006, 78(6):1011-1025.

21. Jimenez-Sanchez G, Childs B, Valle D: Human disease genes. Nature 2001, 409(6822):853-855.

22. Wetlaufer DB: Nucleation, rapid folding, and globular intrachain regions in proteins. Proc Natl Acad Sci USA 1973, 70(3):697-701.

23. Patthy L: Modular assembly of genes and the evolution of new functions. Genetica 2003, 118(2):217-231.

24. Gilbert W: Genes-in-pieces revisited. Science 1985, 228(4701):823-824.

25. Doolittle RF: The multiplicity of domains in proteins. Annu Rev Biochem 1995, 64(1):287-314.

26. Pruitt KD, Tatusova T, Maglott DR: NCBI reference sequences (RefSeq): a curated non-redundant sequence database of genomes, transcripts and proteins. Nucleic Acids Res 2007, 35(suppl_1):D61-65.

27. Harrison P, Kumar A, Lang N, Snyder M, Gerstein M: A question of size: the eukaryotic proteome and the problems in defining it. Nucleic Acids Res 2002, 30(5):1083-1090.

28. Humphery-Smith I: A human proteome project with a beginning and an end. Proteomics 2004, 4(9):2519-2521.

29. Bateman A, Birney E, Cerruti L, Durbin R, Etwiller L, Eddy SR, GriffithsJones S, Howe KL, Marshall M, Sonnhammer ELL: The Pfam protein families database. Nucleic Acids Res 2002, 30(1):276-280.

30. Finn RD, Tate J, Mistry J, Coggill PC, Sammut SJ, Hotz H-R, Ceric G, Forslund K, Eddy SR, Sonnhammer ELL, Bateman A: The Pfam protein families database. Nucleic Acids Res 2008, 36(suppl_1):D281-288.

31. Chothia C: One thousand families for the molecular biologist. Nature 1992, 357(6379):543-544

32. George RA, Heringa J: Protein domain identification and improved sequence similarity searching using PSI-BLAST. Proteins: Struct, Funct, Genet 2002, 48(4):672-681.

33. Wellcome Trust Case Control Consortium: Genome-wide association study of 14,000 cases of seven common diseases and 3,000 shared controls. Nature 2007, 447(7145):661-678.

34. Barrett JC, Cardon LR: Evaluating coverage of genome-wide association studies. Nat Genet 2006, 38(6):659-662.

35. Hamosh A, Scott AF, Amberger J, Bocchini C, Valle D, McKusick VA: Online Mendelian Inheritance in Man (OMIM), a knowledgebase of human genes and genetic disorders. Nucleic Acids Res 2002, 30(1):52-55.

36. Raychaudhuri S, Plenge RM, Rossin EJ, Ng ACY, Purcell SM, Sklar P, Scolnick EM, Xavier RJ, Altshuler D, Daly MJ, International Schizophrenia C: Identifying relationships among genomic disease regions: predicting genes at pathogenic SNP associations and rare deletions. PLOS Genet 2009, 5(6):e1000534.

37. Duncan D, Prodduturi N, Zhang B: WebGestalt2: an updated and expanded version of the Web-based Gene Set Analysis Toolkit. BMC Bioinformatics 2010, 11(Suppl 4):P10.

38. Miretti MM, Walsh EC, Ke $X$, Delgado $M$, Griffiths $M$, Hunt $S$, Morrison J, Whittaker P, Lander ES, Cardon LR, Bentley DR, Rioux JD, Beck S, Deloukas P: A high-resolution linkage-disequilibrium map of the human major histocompatibility complex and first generation of tag single-nucleotide polymorphisms. Am J Hum Genet 2005, 76(4):634-646.

39. Ballouz S, Liu J, Oti M, Gaeta B, Fatkin D, Bahlo M, Wouters M: Application of Gentrepid as a discovery tool to a genome-wide association study.

40. Turner FS, Clutterbuck DR, Semple CAM: POCUS: mining genomic sequence annotation to predict disease genes. Genome Biol 2003, 4(11): R75.

41. The UniProt Consortium: The Universal Protein Resource (UniProt) 2009. Nucleic Acids Res 2009, 37(suppl_1):D169-174.

42. George RA, Spriggs RV, Bartlett GJ, Gutteridge A, MacArthur MW, Porter CT, Al-Lazikani $B$, Thornton JM, Swindells MB: Effective function annotation through catalytic residue conservation. Proc Natl Acad Sci USA 2005, 102(35):12299-12304.

43. Marchini J, Howie B, Myers S, McVean G, Donnelly P: A new multipoint method for genome-wide association studies by imputation of genotypes. Nat Genet 2007, 39(7):906-913.

44. Armitage $P$ : Tests for linear trends in proportions and frequencies. Biometrics 1955, 11(3):375-386.

45. Gabriel S, Schaffner S, Nguyen H, Moore J, Roy J, Blumenstiel B, Higgins J, DeFelice M, Lochner A, Faggart M: The structure of haplotype blocks in the human genome. Science 2002, 296(5576):2225-2229. 
46. Maston G, Evans $\mathrm{S}$, Green M: Transcriptional regulatory elements in the human genome. Annu Rev Genomics Hum Genet 2006, 7:29-59.

47. Nobrega MA, Ovcharenko I, Afzal V, Rubin EM: Scanning human gene deserts for long-range enhancers. Science 2003, 302(5644):413.

48. Ihaka R, Gentleman R: R: A language for data analysis and graphics. J Comput Graph Stat 1996, 5(3):299-314.

49. Ashburner M, Ball CA, Blake JA, Botstein D, Butler H, Cherry JM, Davis AP, Dolinski K, Dwight SS, Eppig JT, Harris MA, Hill DP, Issel-Tarver L, Kasarskis A, Lewis S, Matese JC, Richardson JE, Ringwald M, Rubin GM, Sherlock G: Gene Ontology: tool for the unification of biology. Nat Genet 2000, 25(1):25-29.

50. Kapushesky M, Emam I, Holloway E, Kurnosov P, Zorin A, Malone J,

Rustici G, Williams E, Parkinson H, Brazma A: Gene expression atlas at the European bioinformatics institute. Nucleic Acids Res 2010, 38(suppl 1): D690-D698.

doi:10.1186/1471-2156-12-98

Cite this article as: Ballouz et al:: Analysis of genome-wide association study data using the protein knowledge base. BMC Genetics 2011 12:98.

\section{Submit your next manuscript to BioMed Central} and take full advantage of:

- Convenient online submission

- Thorough peer review

- No space constraints or color figure charges

- Immediate publication on acceptance

- Inclusion in PubMed, CAS, Scopus and Google Scholar

- Research which is freely available for redistribution

Submit your manuscript at www.biomedcentral.com/submit
C Biomed Central 